\title{
The effect of air bearing contour design on thermal pole-tip protrusion
}

\author{
Joerg Fritzsche $\cdot$ Hui Li $\cdot$ Hao Zheng $\cdot$ \\ Kensuke Amemiya $\cdot$ Frank E. Talke
}

Received: 25 August 2010/Accepted: 23 December 2010/Published online: 11 January 2011

(C) The Author(s) 2011. This article is published with open access at Springerlink.com

\begin{abstract}
Thermal flying height control has recently been implemented in magnetic recording disk drives to reduce the flying height at the read/write element of magnetic recording sliders. This paper investigates the effect of air bearing contour design on thermal pole-tip protrusion and flying characteristics of magnetic recording sliders. A number of air bearing surface designs are examined to study the relationship between air bearing surface design and efficiency of thermal pole-tip protrusion.
\end{abstract}

\section{Introduction}

Thermal flying height control (TFC) sliders have recently been implemented in magnetic recording disk drives (Suk et al. 2005; Kurita et al. 2005). Thermal flying height control sliders use a small resistance heater incorporated

J. Fritzsche $(\bowtie) \cdot$ H. Zheng · F. E. Talke

Center for Magnetic Recording Research,

University of California, 9500 Gilman Drive, La Jolla,

San Diego, CA 92093-0401, USA

e-mail: jfritzsche@ucsd.edu

H. Zheng

e-mail: hao@talkelab.ucsd.edu

F. E. Talke

e-mail: ftalke@ucsd.edu

H. Li $\cdot$ K. Amemiya

Storage Mechanics Laboratory, Hitachi Asia Ltd., 7 Tampines Grande, \#08-01 Hitachi Square, Singapore 528736, Singapore

e-mail: hli@has.hitachi.com.sg

K. Amemiya

e-mail: kamemiya@has.hitache.com.sg near the read/write element to change the contour of the slider, thereby controlling the flying height of the slider. The heat conducted from the resistance heater in the slider to the disk surface is a function of the flying height and the air bearing pressure distribution between the slider and the rotating disk (Zhou et al. 2008). In order to increase the efficiency of thermal flying height control sliders it is important to optimize the air bearing design of the air bearing surface (ABS) of the slider.

Juang and Bogy (2007) and Liu et al. (2008) found that a small isolated air bearing trailing pad supporting the read/ write element of the slider improves the thermal actuation efficiency of the TFC slider since it generates little air bearing pressure. A pad structure featuring trenches was proposed by Gonzaga and Liu (2009). The triangular profile of the latter design was found to minimize the pressure build-up over the isolated pad, thereby improving thermal protrusion and actuation efficiency.

This paper investigates the effect of air bearing geometry on the thermal protrusion and the actuation efficiency of magnetic recording sliders with flying heights in the sub$10 \mathrm{~nm}$ range. The effect of the size of the trailing pad, the effect of multiple trailing pads as well as the effect of the size and location of side and leading pad is studied. Design principles are discussed to achieve maximum thermal protrusion and actuation efficiency.

\section{Model and numerical simulation}

Figure 1 illustrates the slider/disk interface including all important parameters. Since the energy dissipated in the thin air bearing film is small compared to the energy conducted to the disk (Zhang and Body 1999), the heat flux between slider and disk can be expressed as 


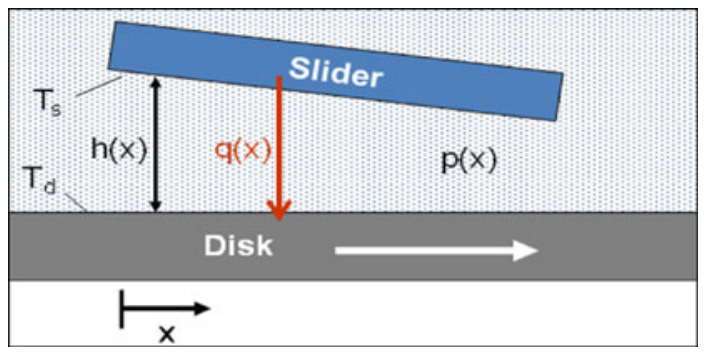

Fig. 1 Slider-disk interface

$q(x)=-k \frac{T_{\mathrm{d}}-T_{\mathrm{s}}}{h(x)+2 \cdot b \cdot \lambda(x)}$

where $k$ is the thermal conductivity of air, while $T_{\mathrm{s}}$ and $T_{\mathrm{d}}$ represent the surface temperature of the slider and the disk, respectively. In eq. $1, h(x)$ is the local spacing between slider and disk and $\lambda(x)$ is the pressure and temperature dependent local mean free path of air. The constant $b$ is given by

$b=\frac{2 \cdot\left(2-\sigma_{\mathrm{T}}\right) \cdot \gamma}{\sigma_{\mathrm{T}} \cdot(\gamma+1) \cdot \operatorname{Pr}}$

where $\gamma$ is the specific heat capacity ratio, $\sigma_{\mathrm{T}}$ is the thermal accommodation coefficient and Pr is the Prandtl number. Chen et al. (2009) proposed an improved and validated model recently. Further models to calculate the heat flux between slider and disk can be found in Zhou et al. (2008).

To perform the numerical analysis, an "automated" simulation code was developed consisting of the following steps. First, the air bearing contour was modeled and meshed. Thereafter, the pressure distribution and the steady-state flying height were obtained using a finite element solution of the Reynolds equations (Wahl et al. 1996). In the next step, the heat transfer coefficient was calculated. The results from this calculation were used as boundary condition in the finite element thermal-structure simulation, providing temperature, and thermal deformation of the slider. Using the information about the deformed slider, we re-calculated the pressure distribution and flying height, determined the heat transfer coefficient again and re-calculated the thermal deformation of the slider. This process was repeated until convergence was achieved $\mathrm{Li}$ et al. (2009). The basic simulation procedure is illustrated in Fig. 2.

\section{Results and discussion}

\subsection{Reference design}

Design-A in Fig. 3 is the basis for all air bearing surface designs investigated in this paper. This design is a sub-

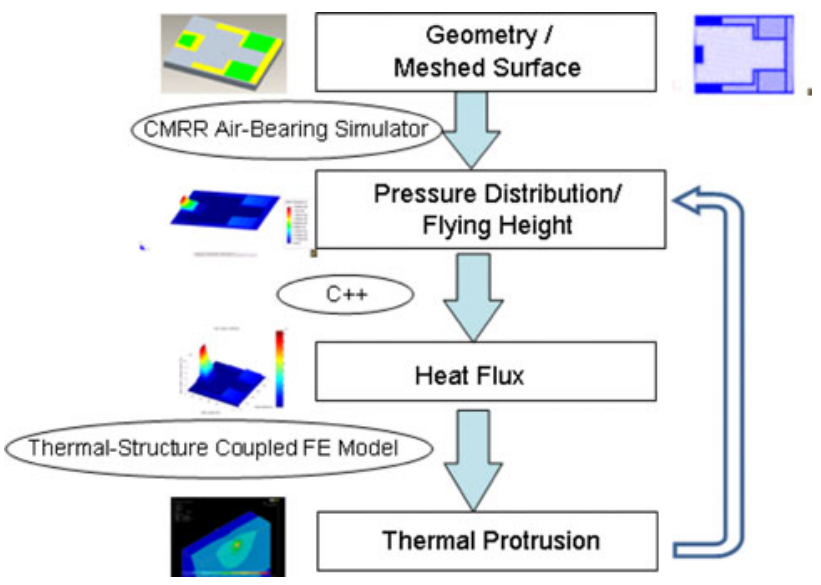

Fig. 2 Basic simulation procedure

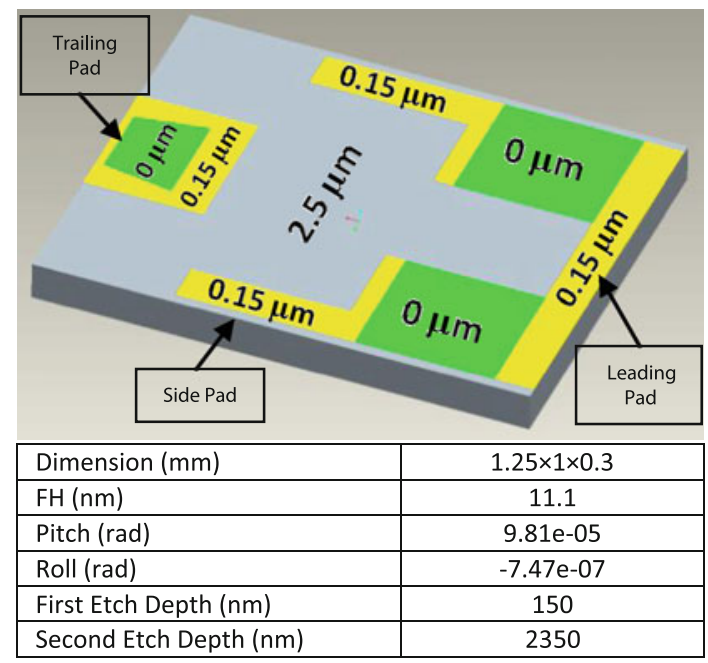

Fig. 3 Reference air bearing surface design (Design-A)

ambient tri-pad air bearing design consisting of one leading pad, two side pads and one trailing pad. The trailing pad contains the read/write elements and the heating coil. In all air bearing surface designs presented, the type of shading and the color used correspond to a particular etch depth of the respective surface measured from the trailing pad. In particular, the yellow and gray surfaces refer to an etch depth from the trailing pad of 0.15 and $2.5 \mu \mathrm{m}$, respectively.

Figure 4 shows the pressure distribution and the heat transfer coefficient for the air bearing surface Design-A without and with thermal protrusion. We observe that both the pressure and the heat transfer coefficient are elevated at the read/write element after activation, i.e. both values are approximately $40 \%$ higher than in the case where no activation is present. Clearly, the reason for this increase is a consequence of the contour change due to the thermal change of the geometry at the read/write element. The 


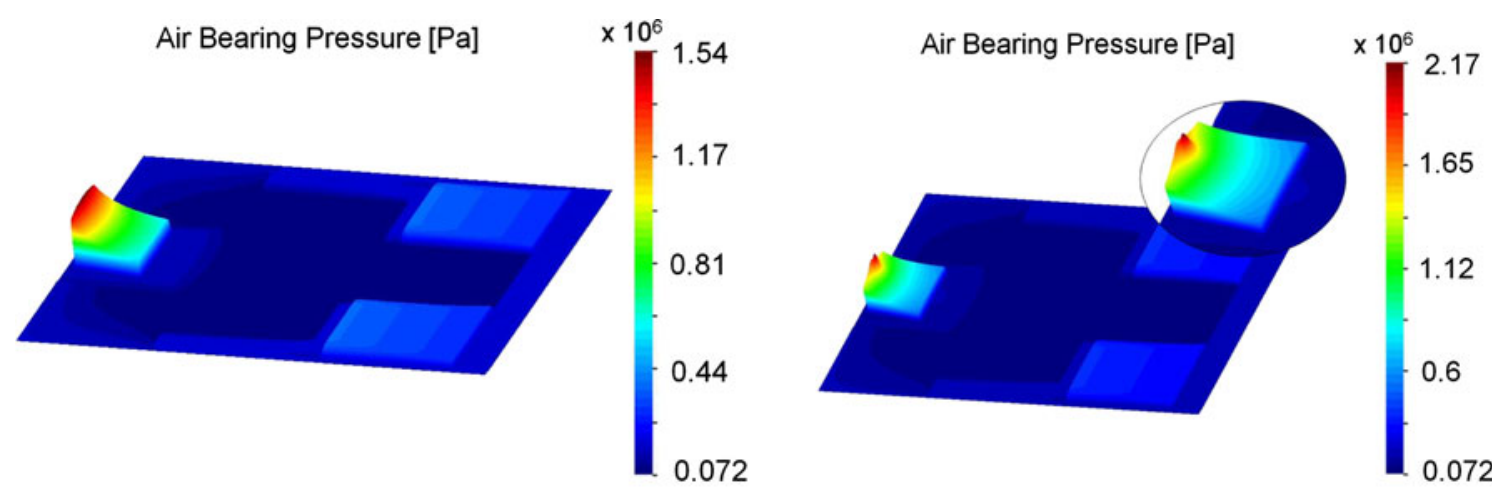

(a) Air bearing pressure
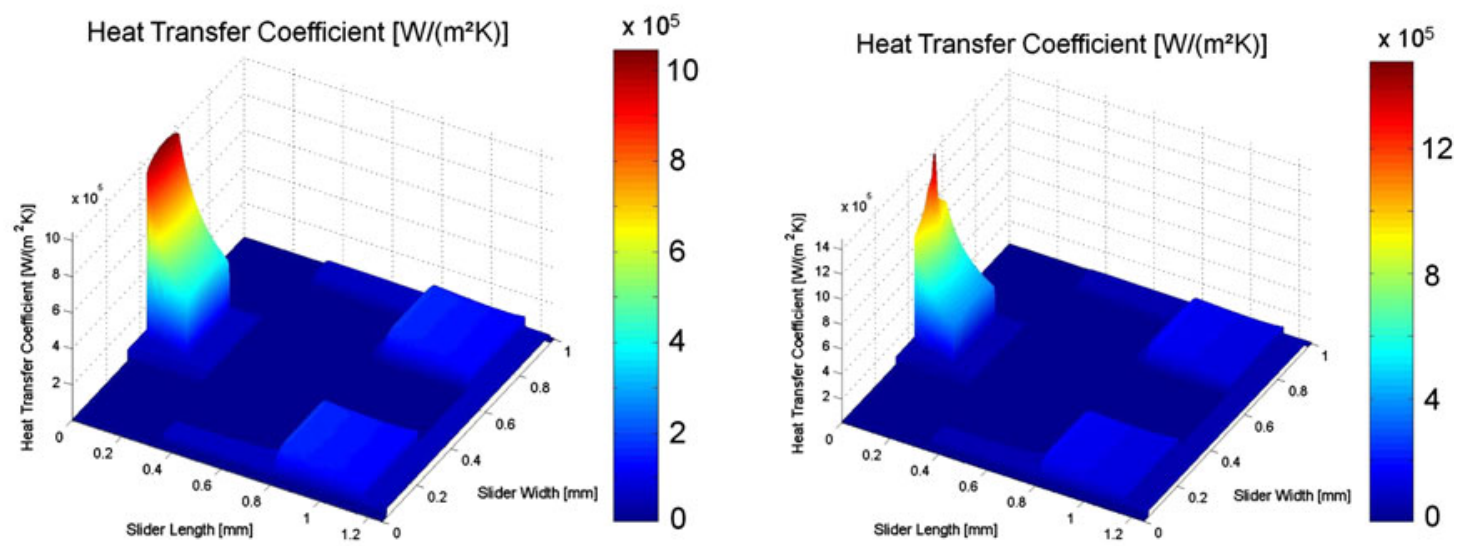

(b) Heat transfer coefficient

Fig. 4 Distribution of $\mathbf{a}$ air bearing pressure and $\mathbf{b}$ heat transfer coefficient before/after protrusion (Design-A)

thermal displacement causes a pressure increase and flying height reduction, which causes the heat transfer coefficient to increase. As a result, the heat loss from the slider to the disk is increased.

Figure 5 shows the center line flying height profile before and after thermal activation. We observe that during thermal activation the flying height is reduced to about $7.9 \mathrm{~nm}$. We also observe that the read/write element is at the lowest point of the slider during thermal protrusion. Compared to the results without thermal activation, where the trailing edge of the trailing pad is the lowest point, this result shows a significant improvement, i.e. the flying height of the read/write element has decreased from about $13 \mathrm{~nm}$ to about $7.9 \mathrm{~nm}$ corresponding to a flying height reduction $(\Delta \mathrm{FH})$ of $5.1 \mathrm{~nm}$.

In evaluating the change of the heat transfer coefficient, we note that a large heat transfer coefficient at the read/ write element is undesirable since high heat transfer coefficients reduce the temperature of the slider and therefore the efficiency of the thermal activation. Equation 1 reveals that the heat flux decreases with decreasing air bearing pressure, since the local mean free path $\lambda(x)$ is inversely proportional to the local air bearing pressure $p(x)$. In order to improve the thermal deformation it is important to find

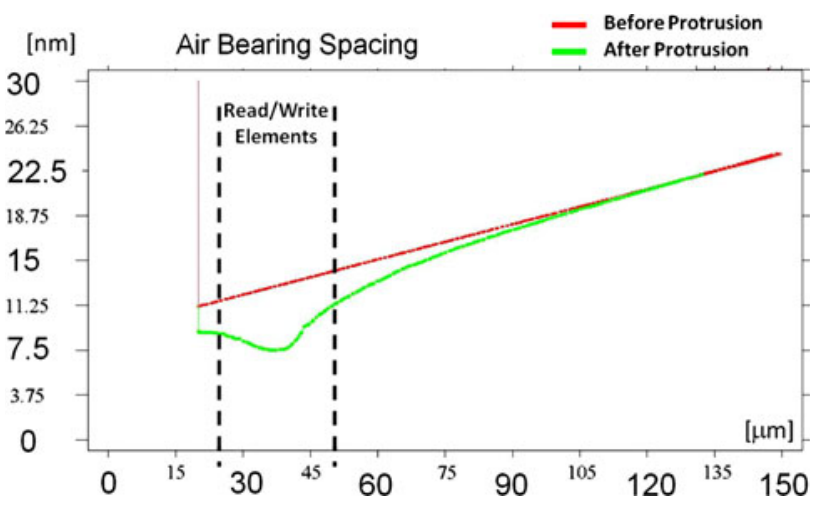

Fig. 5 Center line flying height profile of tailing pad before/after protrusion

air bearing surface designs that reduce the pressure at the $\mathrm{read} / \mathrm{write}$ element and thus reduce the heat flux between slider and disk.

\subsection{Additional trailing pad}

Design-B shows a different layout with an 'additional trailing pad' [see Fig. 6a (red pad)]. The original trailing 
Fig. 6 Slider ABS designs and their air bearing pressure distribution
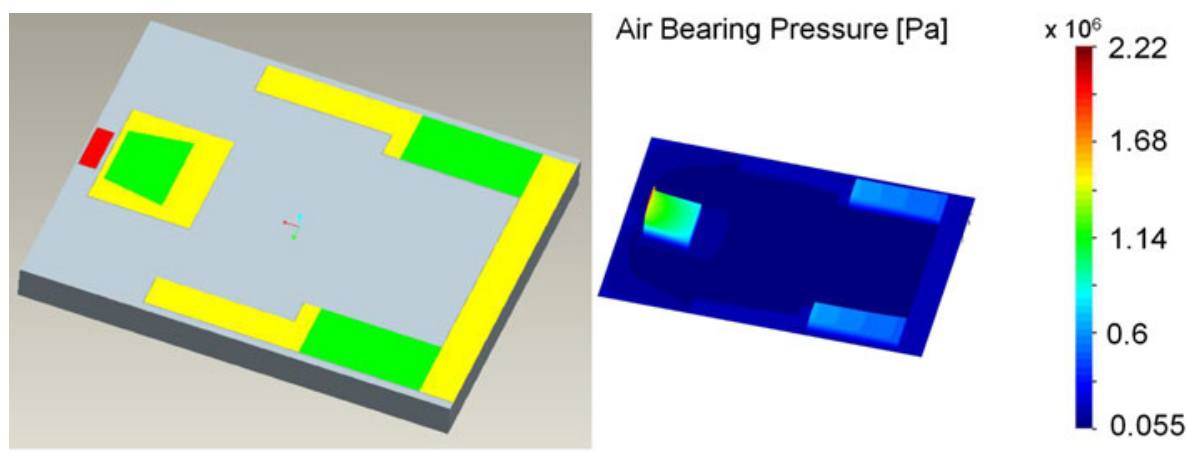

(a) Design-B
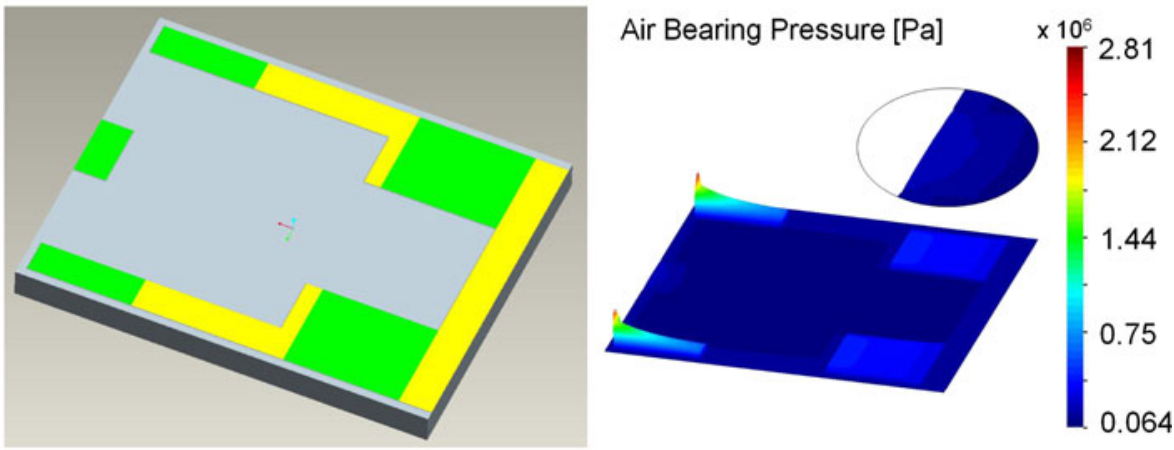

(b) Design-C
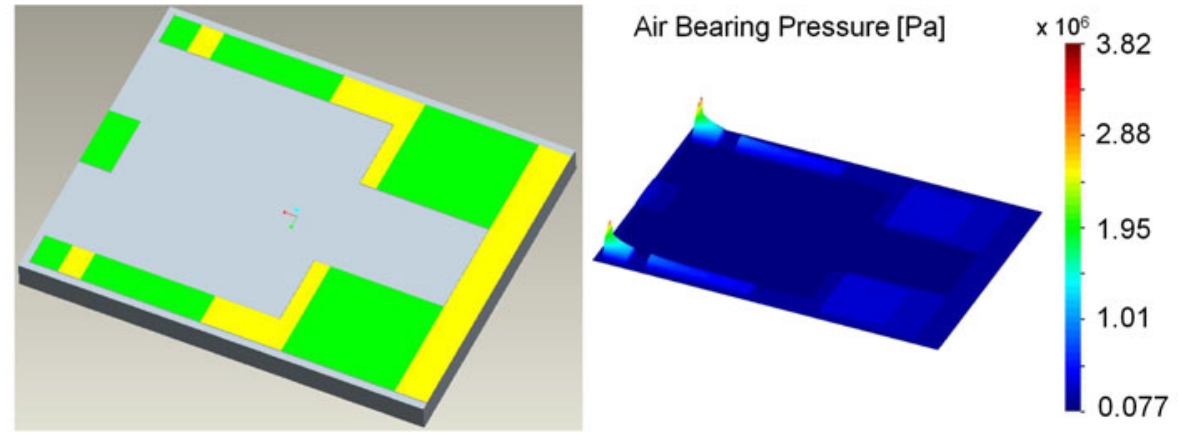

(c) Design-D

pad is moved upstream and an additional pad is added close to the trailing edge. This additional pad is just large enough to house the read/write elements and the heater. Furthermore, the size of the leading pad is reduced to decrease the pitch angle of the slider.

As a result of this change one can see that the pressure increase at the trailing pad prior to thermal activation is similar to that of Design-A. However, the pressure increase at the additional pad is very small. Since the additional pad carries the read/write element, we note that the pressure at the read/write element is reduced to about $90 \%$ of the value of Design-A even though the overall maximum pressure is found to increase by about 20\%. Compared to Design-A, we observe from Fig. 7a that the heat transfer coefficient is reduced by about $80 \%$ at the read/write element. At the same time the flying height is reduced by about $4.5 \mathrm{~nm}$.

The addition of an isolated small pad, as shown in Design- $\mathrm{B}$, represents one way to reduce the pressure at the $\mathrm{read} /$ write element. However, the improved performance for thermal pole-tip control is obtained at the expense of the ability of the slider to follow disk waviness. In particular, since the slider in Design-B is supported by a pad that does not carry the read/write element, this design is not ideal with respect to following the disk waviness. Disturbances in the disk surface will likely make contact with the low-pressure additional pad at the trailing edge, thereby causing increased flying height modulation at the read/ write element. This is a potential disadvantage especially if 
the flying height is reduced to about $3 \mathrm{~nm}$ due to the increased risk of slider-disk contacts.

\subsection{Long side pads}

Design-C uses a different approach to reduce the pressure by introducing long side pads. The side pads are extended to the trailing edge and the size of the leading pad is decreased to lower the flying height. The size of the trailing pad is reduced but is still sufficiently large to support the $\mathrm{read} / \mathrm{write}$ elements.

This design produces a different pressure profile. On the long side pads the pressure is building up to a maximum value of about $2.8 \mathrm{e}+6 \mathrm{~Pa}$ which is twice the maximum pressure of Design-A. The reason for this high pressure is related to the lower flying height of the side pads compared to the trailing pad of Design-A. As a result the flying height of the trailing pad is reduced to about $9.3 \mathrm{~nm}$.

It is import to look at the pressure distribution (Fig. 6b). The pressure can build up on the side pads over a distance of the order of the length of the slider. The trailing pad, on the other hand, is short compared to the length of the side pads. Thus, there is less distance for the pressure to build up. The low pressure will cause a low value of the heat transfer coefficient at that location. As expected, Fig. 7b shows a low heat transfer coefficient at the read/write element and a high heat transfer coefficient at the side pads. Again, the important location to look at for the TFC slider is the location of the read/write element. The heat transfer coefficient is about $75 \%$ lower compared to Design-A and comparable to that for Design-B. Design-C is less affected by flying height modulations compared to Design-A since the supporting high pressure peaks of the side pads are

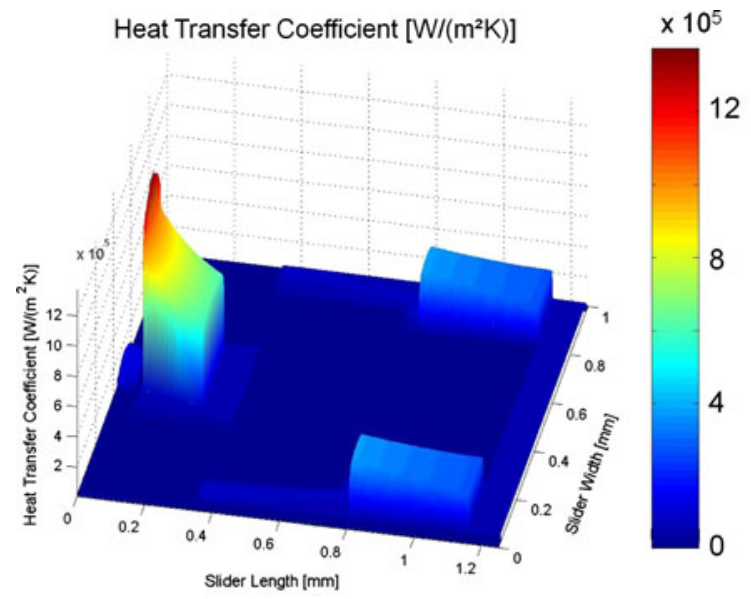

(a) Design-B

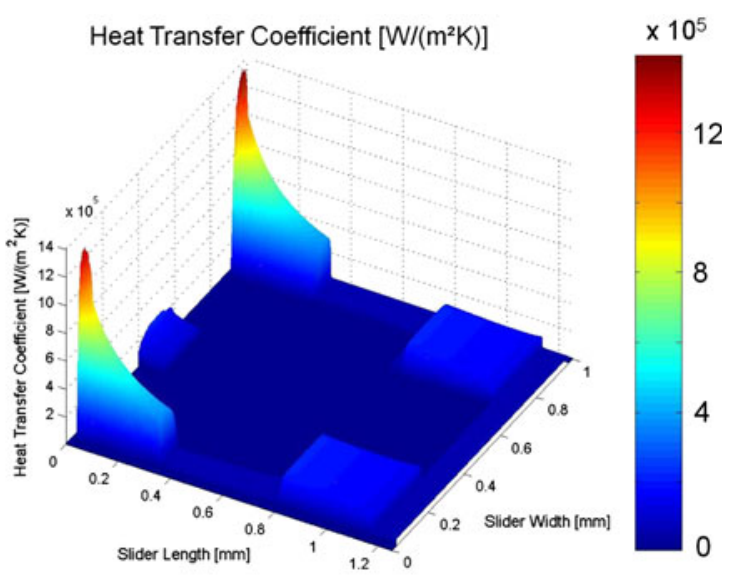

(b) Design-C

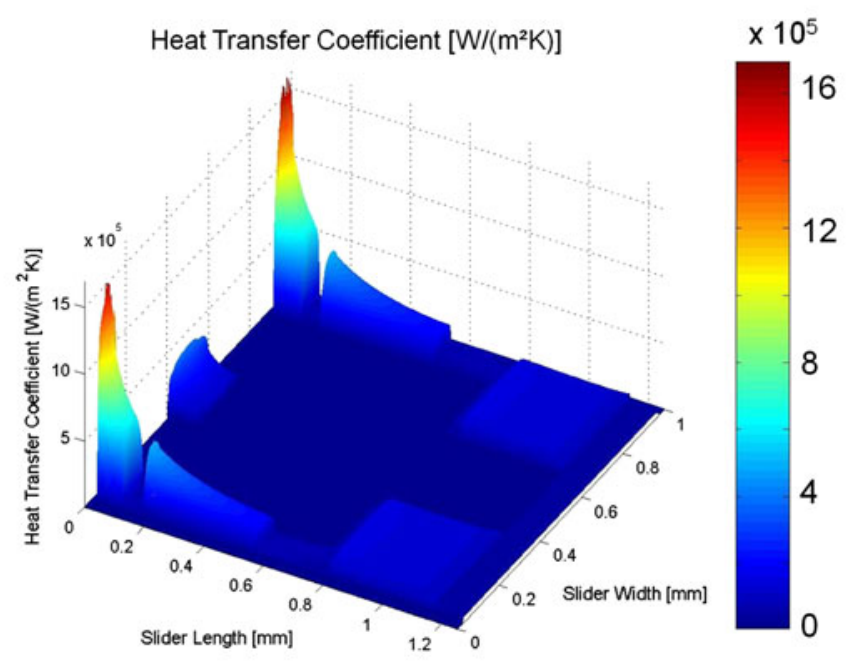

(c) Design-D

Fig. 7 Distribution of the heat transfer coefficient of ABS designs in Fig. 6 
located at the same distance from the leading edge as the $\mathrm{read} / \mathrm{write}$ element is.

Design-D is very similar to Design-C. The main difference is that the side pads are separated into two different sections by introducing a gap close to the trailing edge. The intention of implementing this gap was to investigate whether the location of the pressure peaks can be varied by adding gaps to the pads. We observe from Fig. $6 c$ that the gaps have only a small effect on the pressure at the trailing pad although they caused a reduction in the flying height by about $2 \mathrm{~nm}$ compared to Design-C.

To reduce the effect of roll motion during slider-disk contacts, it seems desirable to move the pressure peaks of the side pads as close as possible to the trailing pad without increasing the pressure at the read/write element.

\subsection{Additional middle pads}

In Design-E the side pads of the previous designs are removed and two middle pads are added right next to the trailing pad (Design-E1). To keep the advantages of Design-C and Design-D, the middle pads end at the trailing edge of the slider. In this case the pressure at the trailing pad shows a higher value than at the middle pads. This is not a good approach to reduce the heat transfer coefficient. Compared to former designs the flying height is relatively high. This is the reason for the low maximum pressure for this design.

For Design-E2 the leading pad size is reduced and the middle pad is made narrower and longer to decrease the flying height. We observe that the trailing pad is the location with the highest pressure peak and that the shape of the pressure peak has changed. In particular, we do not observe a single maximum in the center of the trailing pad but rather two maxima that are located at the outside regions of the trailing pad. In fact, the longer middle pads seem to shift the position of the maximum pressure. Figure $9 \mathrm{~b}$ shows that we also observe two maxima for the heat transfer coefficient.

From Design-E2 we have observed that an increase in the length of the middle pads moves the pressure peak away from the trailing pad. We have therefore extended the middle pads in Design-E3 all the way to the leading pad to see how this design modification affects the pressure distribution. We observe that the flying height of the slider is about $4.2 \mathrm{~nm}$. The high pressure peaks have moved to the middle pads and the pressure at the trailing pad is reduced (Fig. 8c). Furthermore, the overall maximum pressure is reduced even though the flying height is decreased. The heat transfer coefficient shows a similar behavior with a relatively low heat transfer coefficient at the trailing pad and a higher one at the middle pads (Fig. 9c).
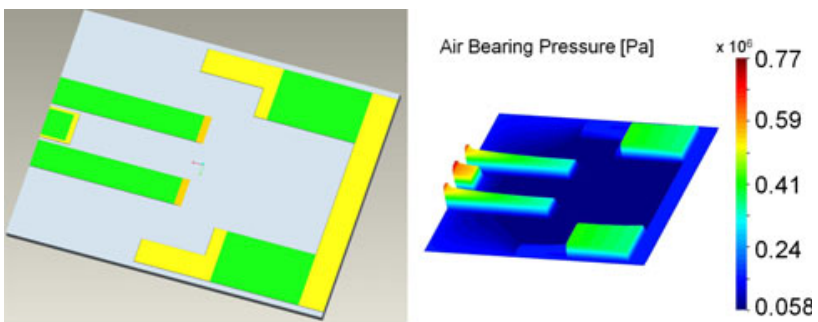

(a) Design-E1

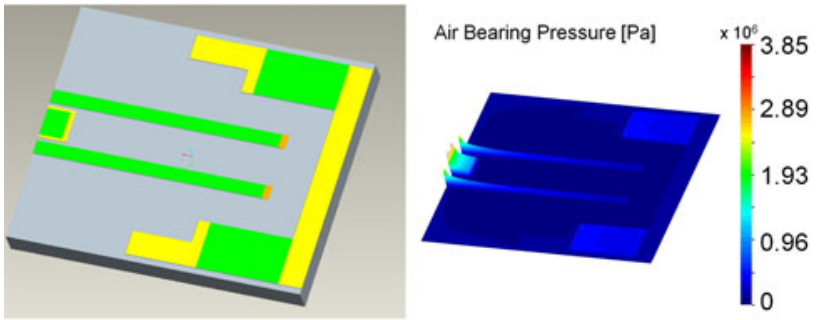

(b) Design-E2

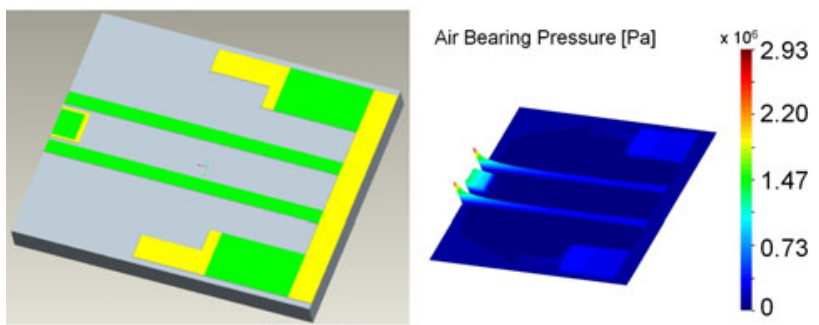

(c) Design-E3

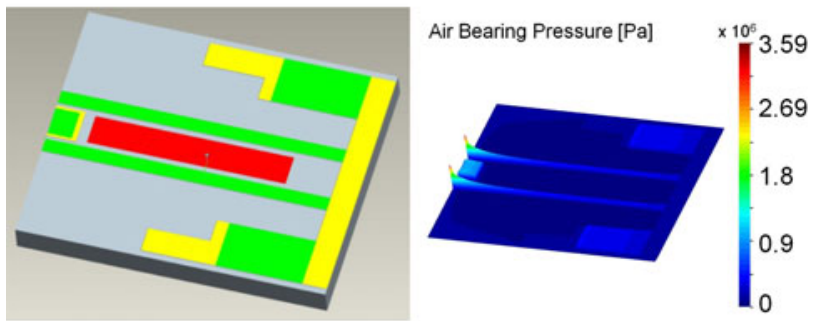

(d) Design-E4

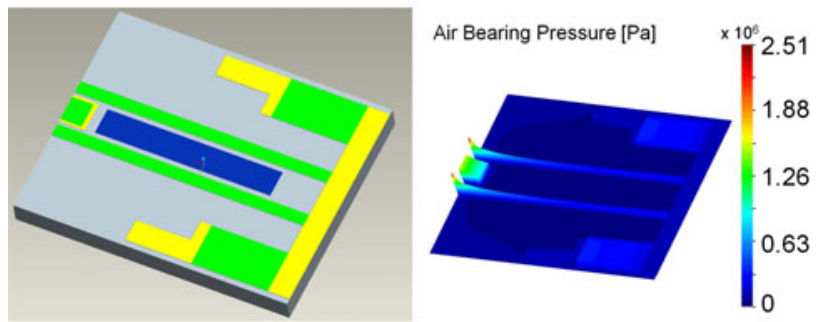

(e) Design-E5

Fig. 8 Slider ABS designs and their air bearing pressure distribution

From Design-E1/E2/E3 one can conclude that an increased length of the middle pads will decrease the pressure at the trailing pad. On the other hand, decreasing the length will cause a pressure increase at the trailing pad. 


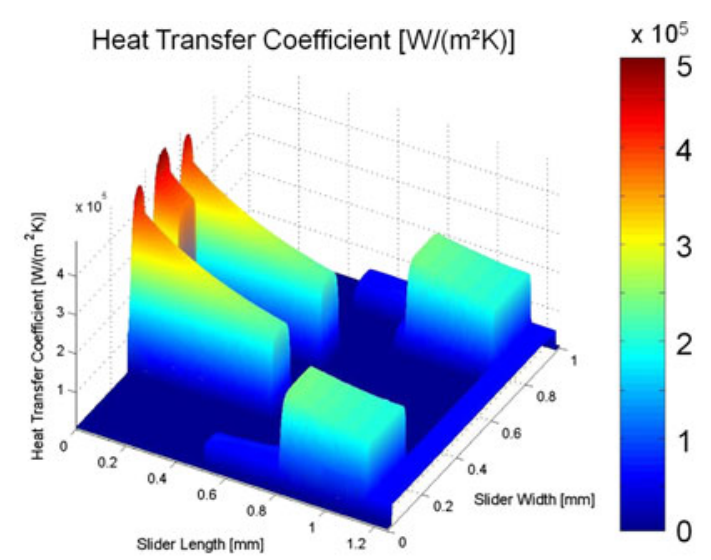

(a) Design-E1

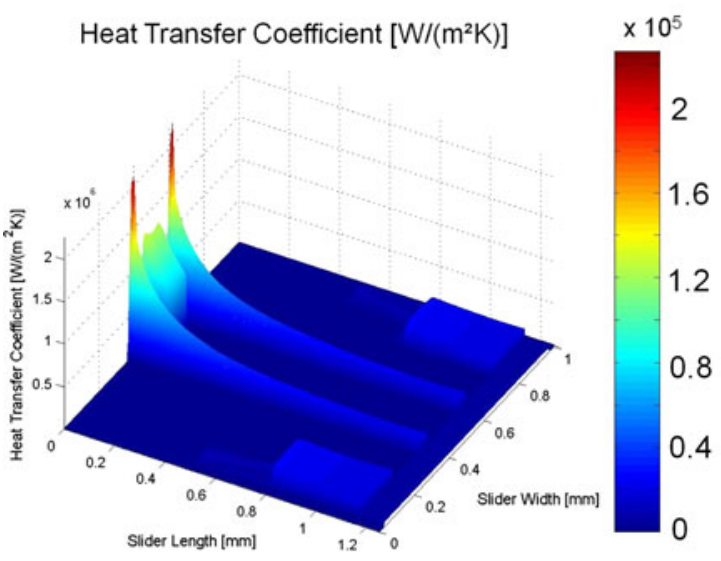

(c) Design-E3

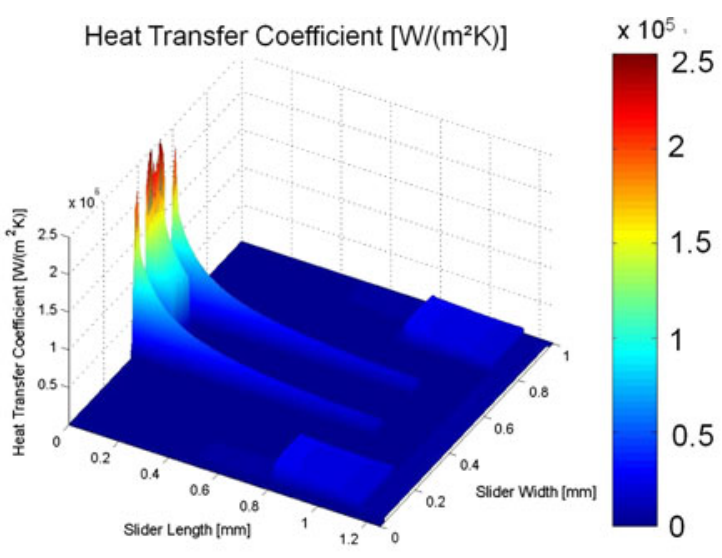

(b) Design-E2

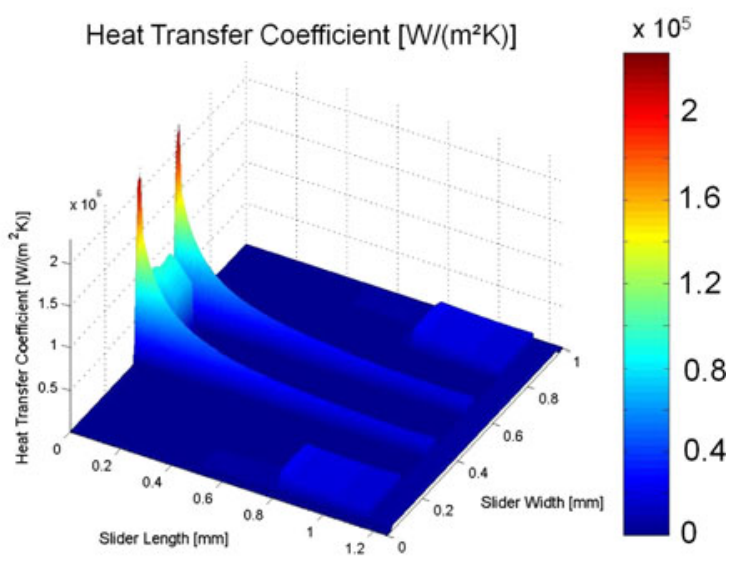

(d) Design-E4

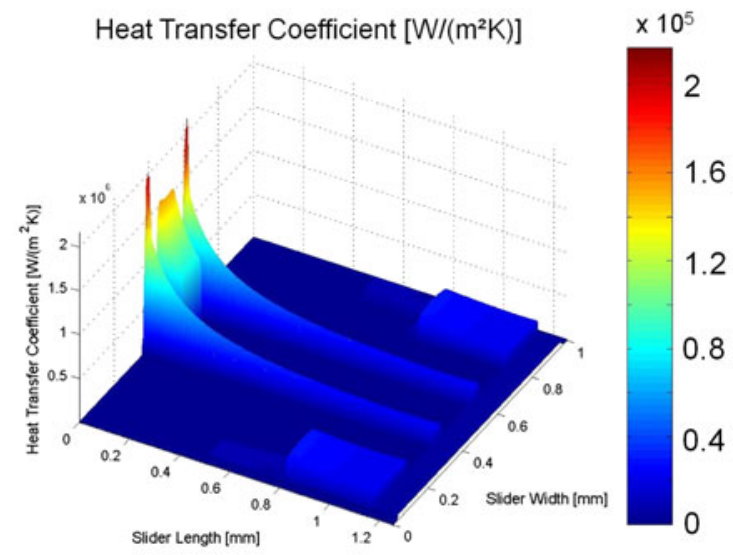

(e) Design-E5

Fig. 9 Distribution of the heat transfer coefficient of ABS designs in Fig. 9

\subsection{Center recess/pad}

The designs in this section will focus on ways to further decrease the pressure at the read/write element. Design-E4 is based on Design-E3. A recess of $300 \mathrm{~nm}$ depth is added in the center between the middle pads (see Fig. 8d, red area).
The recess shifts the pressure from the trailing pad to the middle pads. At the middle pads the pressure is increased compared to Design-E3 but at the trailing pad it is decreased. At the same time the flying height is increased by about $1.2 \mathrm{~nm}$. The question arises whether the pressure drop at the trailing pad is due to the increased flying height. Although this is a possible reason, we note that the pressure 
at the middle pads is increased even though the flying height of the middle pads is increased. This fact shows that the increased flying height cannot be the only reason for the pressure drop. In any case, a pressure shift from the trailing pad to the middle pads can be observed. In addition, the heat transfer coefficient (Fig. 9d) at the read/write element is reduced to about $70 \%$ of the value for Design-E3.

Design-E5 is similar to Design-E4, except that the center recess of Design-E4 is modified to a raised pad of $300 \mathrm{~nm}$ height. As a result the opposite effect of Design-E4 can be observed. Compared to Design-E3 the flying height is decreased by about $0.7 \mathrm{~nm}$ and the pressure at the trailing pad is increased. On the other hand, the pressure at the trailing edge of the middle pads is decreased. This implies that in contrast to a recess between the middle pads, a raised pad causes a shift of the pressure to the trailing pad, increasing the heat transfer coefficient at the read/write element (Fig. 9e).

\section{Conclusion}

In this study, the effect of air bearing contour design on the efficiency of thermal activation in TFC sliders was investigated. The results show that the heat flux between the disk and the pad carrying the read/write element should be as small as possible. By changing the layout of the air bearing surface it is possible to move the pressure distribution within the air bearing. Using different pad shapes and pad sizes we have shown that it is possible to reduce the pressure at the read/write element as well as the heat flux coefficient at the same location. We also have shown that location and shape of the pads have major influence on the flying characteristics.

To achieve desirable flying characteristics it is important to place the side pads as close as possible to the read/write element without causing the pressure at the read/write element to increase. Moving the pads as close as possible to the read/write element without causing an increase in the pressure at the read/write element defines the challenge for air bearing designers of TFC sliders. This challenge is equivalent to the trade-off between flying characteristic and energy efficiency of a slider.

Open Access This article is distributed under the terms of the Creative Commons Attribution Noncommercial License which permits any noncommercial use, distribution, and reproduction in any medium, provided the original author(s) and source are credited.

\section{References}

Chen D, Liu N, Bogy DB (2009) A phenomenological heat transfer model for the molecular gas lubrication system in hard disk drives. J Appl Phys 105:084-303

Gonzaga LV, Liu B (2009) Trenched trailing pad air bearing surface structure for improved thermal actuation efficiency. IEEE Trans Magn 45(11):4994-4997

Juang JY, Bogy DB (2007) Air-bearing effects on actuated thermal pole-tip protrusion for hard disk drives. ASME J Tribol 129(3): $570-578$

Kurita M, Xu J, Tokuyama M, Nakamoto K, Saegusa S, Maruyama Y (2005) Flying-height reduction of magnetic-head slider due to thermal protrusion. IEEE Trans Magn 41:3007-3009

Li H, Kurita M, Xu JG, Yoshida S (2009) Iteration method for analysis of write-current-induced thermal protrusion. Microsyst Technol. doi:10.1007/s00542-009-0775-8

Liu B, Yu SK, Zhou WD, Wong CH, Hua W (2008) Low flyingheight slider with high thermal actuation efficiency and small flying-height modulation caused by disk waviness. IEEE Trans Magn 44:145-150

Suk M, Miyake K, Kurita M, Tanaka H, Saegusa S, Robertson N (2005) Verification of thermally induced nanometer actuation of the magnetic recording transducer to overcome mechanical and magnetic spacing challenges. IEEE Trans Magn 41(11): $4350-4352$

Wahl MH, Lee P, Talke FE (1996) An efficient finite element based air bearing simulator for pivoted slider bearings using biconjugate gradient algorithms. ASME/STLE Tribol Trans 39(1):130-138

Zhang S, Body DB (1999) A heat transfer model for thermal fluctuations in a thin slider/disk air bearing. Int $\mathrm{J}$ Heat Mass Transf 42:1791-1800

Zhou WD, Liu B, Yu SK, Hua W, Wong CH (2008) A generalized heat transfer model for thin film bearings at head-disk interface. Appl Phys Lett 92:043109 